\title{
Controlled Attenuation Parameter in Healthy Individuals Aged 8-70 Years
}

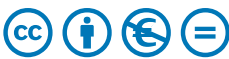

Authors

Anders Batman Mjelle1, 2D, Anesa Mulabecirovic ${ }^{1}$, Edda Jonina Olafsdottir ${ }^{2}$, Odd Helge Gilja1, 3, 4, Roald Flesland Havre2, 3, 4미, Mette Vesterhus 5,6

Affiliations

1 Department of Clinical Medicine, University of Bergen, Bergen, Norway

2 Department of Pediatric and Adolescent Medicine, Haukeland University Hospital, Bergen, Norway

3 Department of Medicine, Haukeland University Hospital, Bergen, Norway

4 National Centre for Ultrasound in Gastroenterology, Haukeland University Hospital, Bergen, Norway

5 Department of Medicine, Haraldsplass Diakonale Sykehus AS, Bergen, Norway

6 Department of Clinical Science, University of Bergen, Bergen, Norway

Key words

Controlled attenuation parameter, Fatty liver index, Liver stiffness, measurement, Non-alcoholic fatty liver disease, ultrasound, methods \& techniques

received 04.12 .2020

revised 16.02 .2021

accepted 21.03.2021

\section{Bibliography}

Ultrasound Int Open 2021; 7: E6-E13

DOI $10.1055 / a-1461-4714$

ISSN 2199-7152

(C) 2021. The Author(s).

This is an open access article published by Thieme under the terms of the Creative Commons Attribution-NonDerivative-NonCommercial-License, permitting copying and reproduction so long as the original work is given appropriate credit. Contents may not be used for commercial purpose, or adapted, remixed, transformed or built upon. (https://creativecommons. org/licenses/by-nc-nd/4.0/)

Georg Thieme Verlag KG, Rüdigerstraße 14,

70469 Stuttgart, Germany
Correspondence

Anders Batman Mjelle

Department of Pediatric and Adolescent Medicine,

Haukeland Universitetssjukehus,

Jonas Lies vei 65

5021 Bergen

Norway

Tel. : +4755975000, Fax : +4755975147

abmjelle@gmail.com

\section{ABSTRACT}

Purpose Controlled attenuation parameter (CAP) is a noninvasive method to assess the presence of liver steatosis. It has been evaluated in children and adults, mainly in either the obese or in subjects with suspected liver disease. Our aim was to describe CAP in healthy non-obese subjects without suspected liver steatosis and to suggest cutoff levels for steatosis.

Materials and Methods We prospectively recruited 187 individuals aged $8-70$ years. All underwent clinical examination, including height and weight measurement. Body mass index (BMI) was calculated and converted into z-scores. To exclude liver pathology, B-mode ultrasound and liver stiffness measurements were performed in all prior to CAP measurement. Blood was drawn for liver biochemistry in adults.

Results CAP was associated with BMI z-score across all ages. CAP started to rise alongside BMI z-score already in subjects with a BMI below average. CAP values were higher in adults than in children $(p<0.001)$, and higher in adult males than adult females $(p=0.014)$. CAP did not correlate with age within the adult or pediatric cohorts. CAP was highly correlated with the fatty liver index. 18 and $23 \%$ of subjects showed CAP above the suggested cutoff value for children and adults, respectively.

Conclusion CAP was correlated with BMI z-score, even in individuals with a below-average BMI . We found CAP above published cutoff values in a substantial proportion of presumably healthy, non-obese children and adults, warranting further research to clarify whether this represents non-obese non-alcoholic fatty liver disease or if reference values need adjustment. 


\section{Introduction}

Non-alcoholic fatty liver disease (NAFLD) has a high impact on the global health burden, with a prevalence of $25 \%$ [1]. Although commonly associated with obese adults, it is the most prevalent liver disease in children [2]. Even in lean adolescents, the prevalence is estimated to be $8 \%$ [3]. In adults, non-obese and lean NAFLD is associated with increased mortality [4].

The criterion standard for diagnosis of NAFLD is liver biopsy. It is often performed in children to stage the severity and to eliminate other liver diseases [2], but its use is more limited in adults [5]. NAFLD can potentially cause fibrosis and cirrhosis, and ultimately chronic liver failure necessitating liver transplantation. Thus, noninvasive monitoring is warranted. Conventional ultrasound (US) examination is applied in both diagnosis and follow-up, with a global sensitivity and specificity of 0.85 and 0.94 , respectively, but it is not recommended due to poor performance in cases of mild steatosis (i. e., involving $5-33 \%$ of hepatocytes) $[2,5,6]$.

Ultrasound attenuates as the US waves penetrate tissue. To compensate for this, the time-gain compensation function is used to amplify reflections from deeper locations to obtain a more even Bmode image. The attenuation increases with increasing fat content in the liver tissue. The measurement of attenuation at different scanning depths and in different organs, e. g., using the hepato-renal index for assessing fatty liver in NAFLD patients, has been described and used to identify and quantify liver steatosis [7].

The controlled attenuation parameter (CAP), implemented in the Fibroscan ${ }^{\circledR}$ system, uses this attenuation within liver tissue to calculate the CAP, simultaneously measuring liver stiffness. CAP has been shown to correlate with the histopathological degree of liver steatosis in adults [8,9] and children [10], with a pooled sensitivity and specificity for mild steatosis of 0.87 and 0.91 , respectively [11]. On this basis, adult guidelines suggest that CAP is a promising tool but state that there is a need for more information [5]. Different cutoff values have been suggested for the diagnosis of NAFLD: for adults $248 \mathrm{~dB} / \mathrm{m}$ [9] and in children $225 \mathrm{~dB} / \mathrm{m}$ [10]. Reliability criteria for CAP measurements are not established, but both the interquartile range (IQR) (e. g., $<0.4)$ and IQR divided by the me$\operatorname{dian}(\mathrm{IQR} / \mathrm{M})($ e. g., $<0.1,<0.2$, or $<0.3$ ) have been proposed $[12,13]$.

There is a lack of studies in pediatric and combined adult and pediatric populations, particularly in subjects without obesity or suspected liver disease. We aimed to explore CAP in healthy nonobese children and adults to establish normal CAP values, with simultaneous examination with B-mode ultrasound and liver stiffness measurements. The study was part of a previously published project aiming to establish reference LSM values for children and adults $[14,15]$.

\section{Materials and Methods}

\section{Subjects}

The study was performed at a single university hospital from August 2017 to January 2018. We prospectively recruited children aged $8-17$ years and adults aged $25-70$ years. All examinations were performed by one of two operators experienced in liver stiffness measurements and certified Fibroscan users. Inclusion criteria consisted of valid CAP and LSM using Fibroscan, no history of liver disease or any chronic disease with the potential of affecting the liver, and lack of obesity defined as an international obesity task force (IOTF) grade 2. A total of 87 children and 100 adults were recruited. Height, weight, waist circumference, and body mass index (BMI) were recorded and converted into z-scores (standard score; standard deviations below or above the mean). BMI z-score was used in analyses in children and in the entire cohort, while BMI was used in analyses with adults. Blood was drawn for liver biochemistry analyses in all adults but not in children. Fatty liver index, non-alcoholic fatty liver disease (NAFLD) fibrosis scores, and Fib-4, a commonly used noninvasive liver fibrosis score, were calculated for all adults using published algorithms [16-18]. Subjects with abnormal liver function tests or B-mode signs of steatosis or splenomegaly were excluded.

\section{B-mode ultrasound evaluation}

All participants underwent B-mode ultrasound evaluation using a standardized protocol, including evaluation of the liver, gall bladder, spleen, and kidneys prior to elastography and CAP measurements. The distance between the skin and the liver capsule was recorded.

\section{Liver stiffness measurements (LSM)}

Elastography and CAP measurements were performed according to the protocol, with subjects in a supine position with the right hand resting under their head, after $\geq 3$ hours of fasting. All participants were examined using transient elastography (TE, Fibroscan with an M-probe), point shear wave elastography (pSWE; in Samsung RS80A with the CA1-7A convex array probe), and twodimensional shear wave elastography (2D-SWE; in children: GE Logiq E9, in adults: GE Logiq S8; both with the C1-6 convex array probe). A valid LSM was defined as the median value of 10 acquisitions, given an interquartile range divided by the median (IQR/M) of $\leq 30 \%$. CAP values were accepted if the LSM by Fibroscan measurement was considered valid.

\section{Controlled attenuation parameter}

Controlled attenuation parameter (CAP) was reported in $\mathrm{dB} / \mathrm{m}$, using the median of 10 acquisitions. The interquartile range (IQR) was registered for all measurements.

\section{Statistical analysis}

We used SPSS version 25 (SPSS Inc, 2016, Armonk, NY) for all analyses. All variables were tested for normality before presenting data as either mean \pm standard deviation (SD) or median (range), as appropriate. For comparison of groups, standard paired T-test, Wilcoxon signed rank test, or Pearson Chi-Square test was used as appropriate. Correlations were tested by Pearson correlation coefficient. The upper limit of normal (ULN) value was defined as mean +1.64 SD unless otherwise specified. P values $<0.05$ were considered significant.

\section{Ethical aspects}

The study was in accordance with the Declaration of Helsinki and approved by the Regional Committee on Medical and Health Research Ethics. 
- Table 1 Background characteristics of patients undergoing ultrasound elastography and controlled attenuation parameter (CAP) measurements.

\begin{tabular}{|c|c|c|c|}
\hline & Total panel & Children & Adults \\
\hline Number & 176 & 82 & 94 \\
\hline Males, number (\%) & $77(43.8)$ & $31(37.8)$ & $46(48.9)$ \\
\hline Age, median (range) & $26(8-69)$ & $13.3(8.4-17.9)$ & $42.5(25-69)$ \\
\hline Body mass index z-score, mean (SD) & $0.2(0.9)$ & $-0.1(1.0)$ & $0.4(0.7)$ \\
\hline Overweight subjects (IOTF=1), n (\%) & 35 (19.9) & $5(6.1)$ & $30(31.9)$ \\
\hline Overweight subjects according to BMI z-score, n (\%) & $22(12.5)$ & $6(7.3)$ & $16(17)$ \\
\hline \multicolumn{4}{|l|}{ Fib-4, n (\%) } \\
\hline Low risk & & - & $70(74.5)$ \\
\hline Indeterminate & & - & $24(25.5)$ \\
\hline High risk & & - & - \\
\hline \multicolumn{4}{|l|}{ NAFLD fibrosis score, $\mathrm{n}(\%)$} \\
\hline Low risk & & - & $94(100)$ \\
\hline Indeterminate & & - & - \\
\hline High risk & & - & - \\
\hline \multicolumn{4}{|l|}{ Fatty liver index, $n$ (\%) } \\
\hline Low risk & & - & 77 (81.9) \\
\hline Indeterminate & & - & $10(10.6)$ \\
\hline High risk & & - & $7(7.4)$ \\
\hline
\end{tabular}

\section{Results}

A total of 176 subjects were included for final analyses ( 82 children aged $8-17$ years [ 31 males; $37.8 \%$ ], 94 adults aged $25-70$ years [46 males; 48.9\%]). Reasons for exclusion were steatosis on B-mode ultrasound in adults $(n=6)$ and either failure to obtain valid measurements $(n=3)$ or obesity $(n=2)$ in the case of children. Background characteristics are shown in $>$ Table 1.

\section{Overall results}

In the total population, the mean CAP was $208 \pm 44.1 \mathrm{~dB} / \mathrm{m}$ (range 100-348).

CAP values increased with age. Dividing participants into children, young adults ( $<39.9$ years), and adults ( $40-70$ years), the mean values were 191,210 , and 232 , respectively ( $p$-values 0.018 , 0.018 , and $<0.001$, respectively, for comparisons between consecutive age groups). In linear regression, CAP was associated with age, BMI z-score, skin-to-capsule distance, and sex ( $\mathrm{p}$-values 0.049 , $0.011,0.011$, and 0.049 , respectively) ( $\triangleright$ Fig. 1). CAP was significantly correlated with BMI z-score in children (rho 0.27, $\mathrm{p}=0.014$ ) and adults (rho 0.54, $\mathrm{p}<0.001$ ). This difference in the degree of correlation reflects a steeper rise in adults than children ( $>$ Fig. 2 ). CAP values were not affected by LSM by either TE, 2D-SWE, or pSWE.

Regarding quality criteria, the overall CAP interquartile range (IQR) was $41 \pm 23$ (range $7-225$ ). We found that $53 \%$ of values were within the proposed quality criteria of IQR $<40$, while 19,55 and $81 \%$ had an IQR/M value less than $0.1,0.2$, and 0.3 , respectively.
IQR exerted no effect on CAP value. IQR was not affected by CAP, LSM (or LSM IQR/M) by any system, sex, or age.

\section{CAP in children}

In children aged 8-17 years, the mean CAP was $191 \pm 38 \mathrm{~dB} / \mathrm{m}$ (range $100-296 \mathrm{~dB} / \mathrm{m}$ ), and $82 \%$ and $94 \%$ of subjects had CAP results within the normal range as defined by the published cutoff value $<225$ for children and $<248 \mathrm{~dB} / \mathrm{m}$ for adults, respectively. ULN calculation yielded a cutoff value of $253 \mathrm{~dB} / \mathrm{m}$. The only significant factors affecting CAP in children were the BMI z-score ( $\triangleright$ Table 3 ) and waist circumference $z$-score (p-values 0.01 and 0.011 , respectively). The skin to capsule distance was not associated with CAP. The CAP value was found to rise with BMI z-score, starting at a BMI z-score between -1 and 0 ( $>$ Fig. 3 ). Using the published cutoff value of $225 \mathrm{~dB} / \mathrm{m}$, BMI z-scores were not significantly higher in the high compared to the low CAP group (mean value +0.34 vs. $-0.2 \mathrm{p}=0.051)$.

CAPIQR was $44.6 \pm 20$ (range 10-101). We found that $45 \%$ had an IQR $<40$, while 9,44 and $72 \%$ had an IQR/M value less than 0.1 , 0.2 , and 0.3 , respectively.

Trying to establish an upper limit of normal (ULN) for these quality criteria in our presumably healthy, non-obese children using the lowest $90 \%$ of values, the IQR and IQR/M cutoff values would be $<69$ and $I Q R / M<0.4$, respectively. 


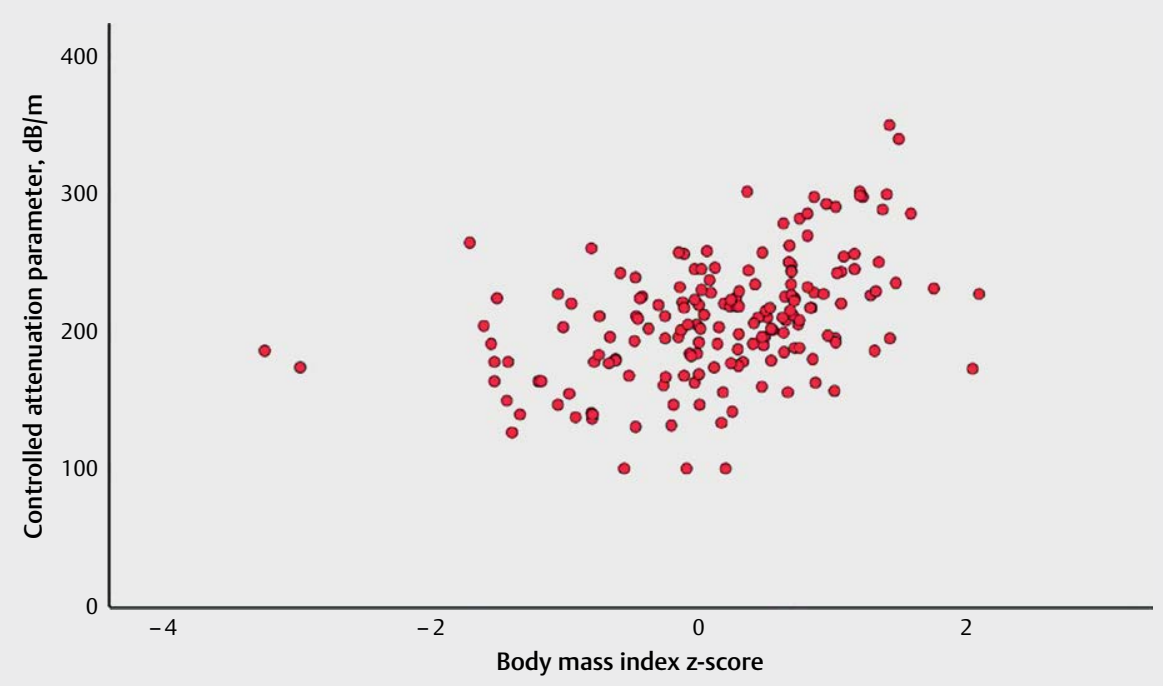

- Fig. 1 Body mass index z-score versus controlled attenuation parameter for all participants, aged 8-70 years. Rho 0.45 ( $p<0.001)$.

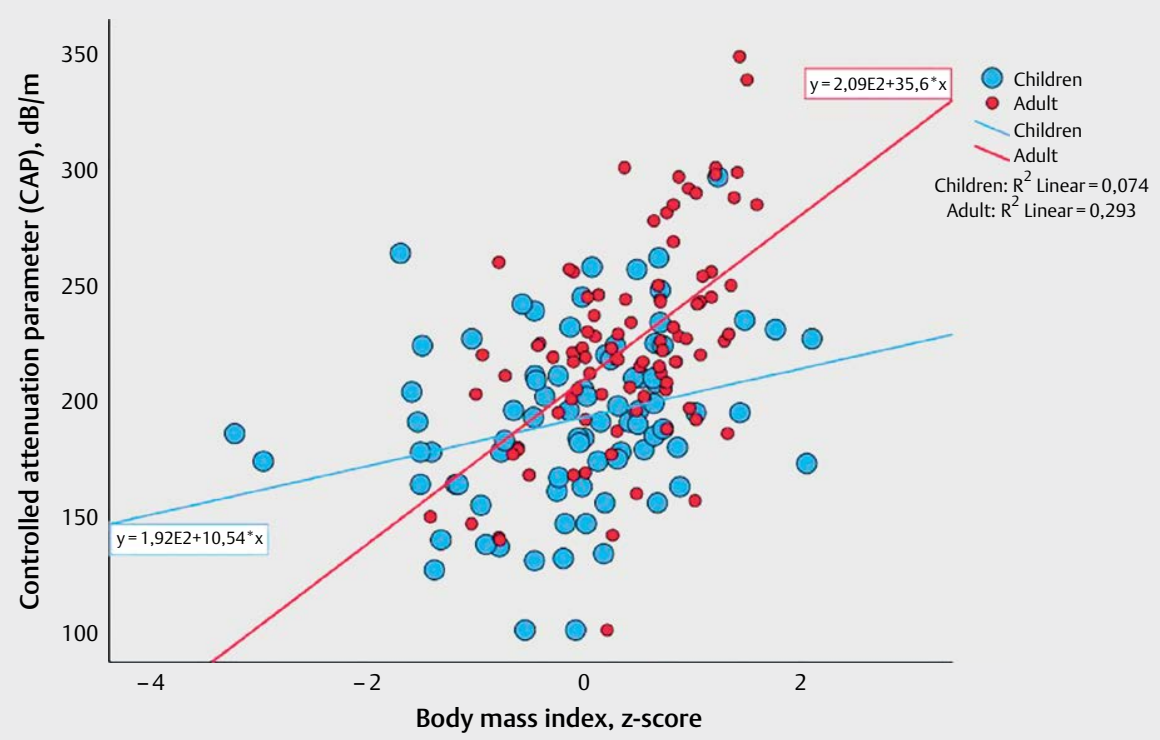

Fig. 2 Body mass index z-score versus controlled attenuation parameter for all participants, with children in blue and adults in red, with fit lines for both subgroups.

\section{CAP in adults}

In adults, the mean CAP was $223 \pm 44 \mathrm{~dB} / \mathrm{m}$ (range 100-348), and $56 \%$ and $77 \%$ of subjects had CAP results within the normal range as defined by the published cutoff value $<225$ for children and $<248 \mathrm{~dB} / \mathrm{m}$ for adults, respectively. ULN calculation yielded a cutoff value of $295 \mathrm{~dB} / \mathrm{m}$. CAP correlated with age, sex, BMI ( $\triangleright$ Fig. 4), waist circumference, and skin to capsule distance ( $\vee$ Table 2 ). However, the skin-to-capsule distance was the only factor showing independent association to CAP in linear regression $(p=0.005)$.

The mean CAP values for men and women were $235 \pm 45$ and $210 \pm 40$, respectively. Adults with CAP > $248 \mathrm{~dB} / \mathrm{m}$ had a signifi- cantly higher BMI compared to those with a lower CAP (mean value 25.8 vs. 23.1, $\mathrm{p}<0.001)$.

CAP values were highly correlated with the fatty liver index (rho $0.55, p<0.001$ ) ( $\triangleright$ Figs. 5 and $\triangleright \mathbf{6}$ ) and correlated with the NAFLD fibrosis score (rho 0.21, $\mathrm{p}=0.046$ ). CAP was not correlated with Fib-4 or ALT ( Table 2). The percentage of subjects with low, indeterminate, or high-risk values for different indexes are shown in - Table 1, based on published values [16, 17, 19, 20].

Interquartile range (IQR) was 37.6 (25), with a range of 7-225. We found that $61 \%$ had an IQR $<40$, while 28,64 , and $89 \%$ had an $\mathrm{IQR} / \mathrm{M}$ value less than $0.1,0.2$, and 0.3 , respectively. 


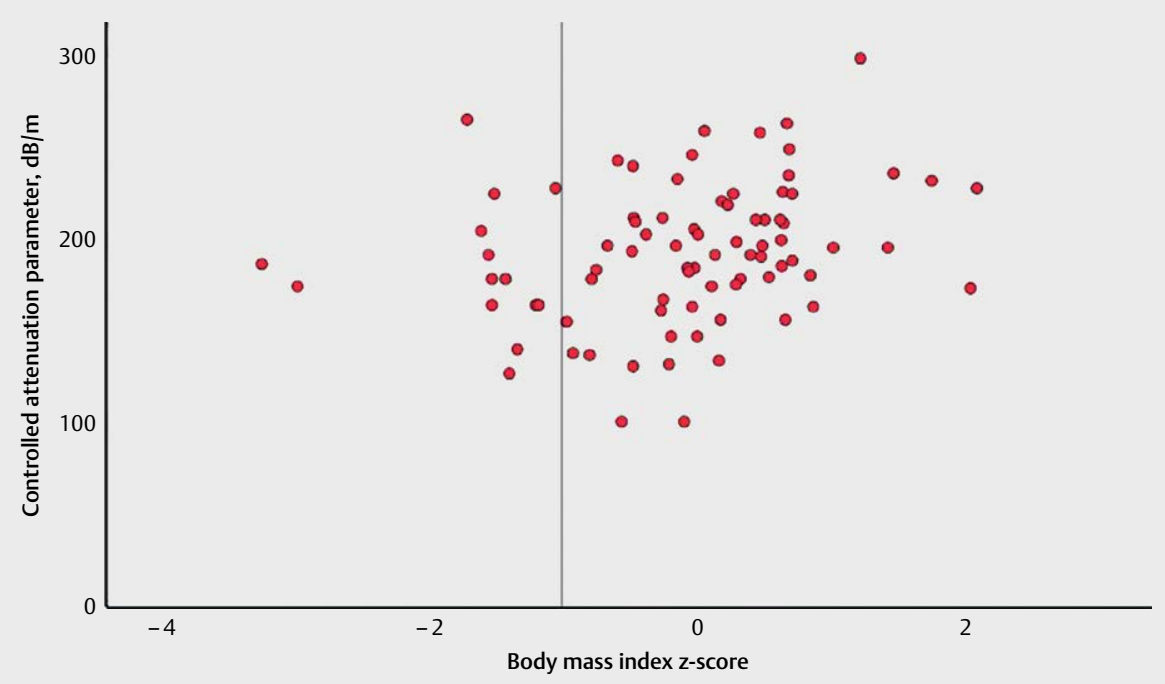

- Fig. 3 Body mass index $z$-score versus controlled attenuation parameter (CAP) for children aged 8-17 years. Rho 0.27 ( $p=0.014)$. The vertical line $(\mathrm{z}$-score -1$)$ reflects where a rise in CAP values begins. Above the -1 reference line, there is a highly significant relationship between CAP and BMI $z$-score (rho 0.36, $p=0.002$ ) while there is no such relationship for those below -1 (rho $-0.04, p=0.88$ ).

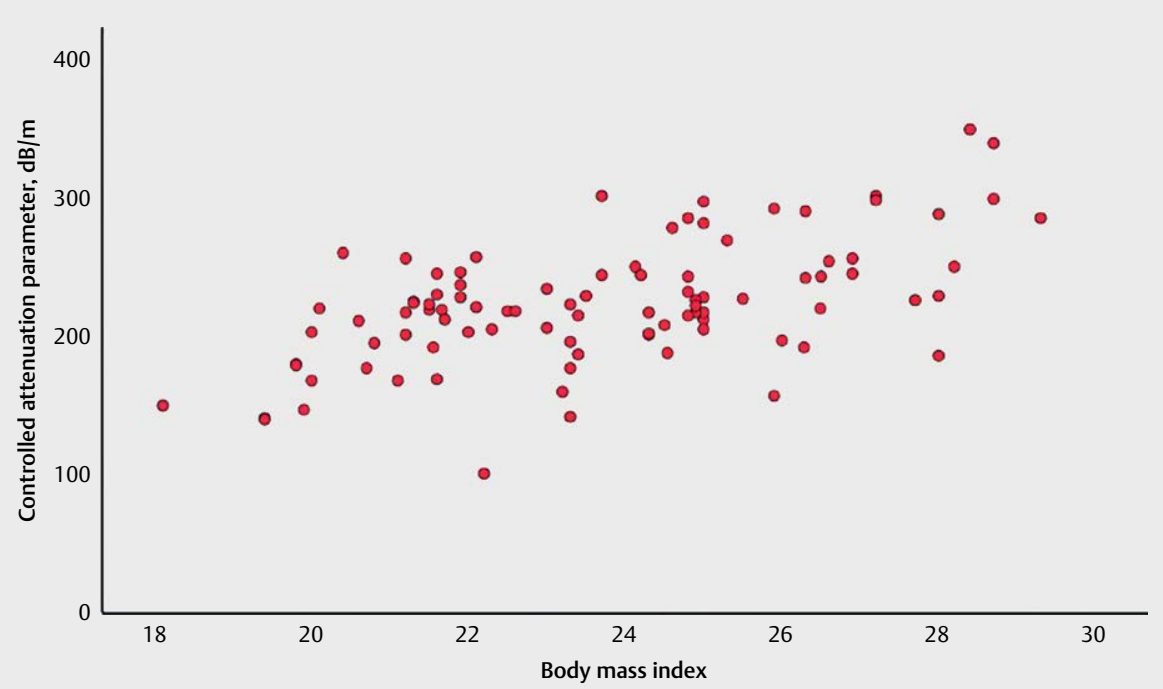

- Fig. 4 Controlled attenuation parameter $(\mathrm{dB} / \mathrm{m})$ versus BMI for adults, demonstrating a significant relationship (rho $0.574, p<0.001)$.

Using the lowest $90 \%$ of values to establish a ULN in adults, the $I Q R$ and IQR/M cutoff values would be $<57$ and $<0.3$, respectively.

\section{Discussion}

This is, to the best of our knowledge, the first study reporting CAP values in healthy non-obese children and adults of all ages. Previous studies in adults have shown that a raised CAP is often found in apparently healthy individuals without evident steatosis upon ultrasound examination [21]. Our study is the first to describe that CAP increases with increasing BMI z-score even in healthy subjects with a BMI z-score below zero (corresponding to a $\mathrm{BMI}<22$ in adults). In line with our findings, an association between CAP and BMI has previously been shown in adults, with an estimated rise of $4.4 \mathrm{~dB} / \mathrm{m}$ per BMI unit. However, whether this is valid for the entire $\mathrm{BMI}$ specter or only for a specific interval remains uncertain $[9,22]$. In contrast, a biopsy-controlled study including children and young adults up to 24 years of age reported a higher BMI in subjects with steatosis than those without steatosis while displaying no significant association between BMI and CAP. All subjects with proven steatosis had a BMI z-score $>1.0$ [10]. 
In children, we found that $18.3 \%$ had a CAP above the suggested cutoff value of $225 \mathrm{~dB} / \mathrm{m}$. Only five children (6.1\%) were above the suggested adult cutoff value of $248 \mathrm{~dB} / \mathrm{m}$; all of them were normal weight, with $B M I$ z-scores ranging from -1.7 to +1.2 . In our study, all but one of the children with a CAP $>248 \mathrm{~dB} / \mathrm{m}$ had BMI $z$-scores $\leq 1.0$, suggesting that steatosis was less likely based on the aforementioned biopsy-controlled study [10]. NAFLD may occur in non-overweight individuals, so-called “lean" NAFLD. A study with 1482 adolescents (12-18 years) with a BMI below the $85^{\text {th }}$ per-

- Table 2 Correlation between CAP and clinical features and laboratory values for adults.

\begin{tabular}{|l|c|c|}
\hline Clinical features & Correlation (r) & p-value \\
\hline Distance from skin to liver capsule & 0.615 & $<0.001$ \\
\hline BMI & 0.574 & $<0.001$ \\
\hline Waist circumference & 0.572 & $<0.001$ \\
\hline Weight & 0.536 & $<0.001$ \\
\hline Waist-to-height ratio & 0.487 & $<0.001$ \\
\hline Fatty liver index & 0.545 & $<0.001$ \\
\hline GGT & 0.324 & 0.001 \\
\hline Triglycerides & 0.298 & 0.003 \\
\hline LDL & 0.270 & $<0.001$ \\
\hline Cholesterol & 0.216 & $<0.001$ \\
\hline HDL & -0.219 & 0.034 \\
\hline NAFLD fibrosis score & 0.207 & 0.046 \\
\hline ALT & 0.173 & n.s. \\
\hline FIB-4 & 0.011 & n.s. \\
\hline
\end{tabular}

BMI: body mass index; GGT: gamma-glutamyl transferase; NAFLD: non-alcoholic fatty liver index; ALT: alanine transaminase; FIB-4: Fibrosis-4; HDL: high-density lipoprotein; LDL: low-density lipoprotein. centile (corresponding to a BMI z-score less than +1 ) found that $8 \%$ had suspected NAFLD, but with no difference in BMI z-score between those with and without suspected NAFLD [3]. Similar or higher incidence rates have been firmly established in lean adults, even in biopsy-proven studies [23-26]. To our knowledge, there are no reports specifically investigating CAP in individuals with a belowaverage BMI.

The rise in CAP across BMI z-scores was steeper in adults than children. Given an identical increase in BMI in children and adults, adults showed a more substantial CAP increase ( $>$ Fig. 2 ).

In adults, CAP was correlated with all anthropometric values indicating obesity: BMI, waist circumference, and skin-to-capsule distance ( $\triangleright$ Table 2 ). These parameters were highly correlated and challenging to use as independent variables. Interestingly, in linear regression, the only factor independently associated with CAP in adults was the skin-to-liver capsule distance, indicating that this measure of subcutaneous fat tissue is more closely associated with steatosis than BMI. BMI is considered a rather reliable predictor of body fat, but obesity may be either overestimated or underestimated based on the amount of muscle tissue, and a high BMI does not necessarily indicate obesity or a high degree of body fat [27]. However, although easy to obtain, skin-to-capsule distance requires ultrasound, making BMI more available for clinicians without ultrasound access.

Our finding of a high degree of correlation between CAP and the fatty liver index confirms previous results [28]. There was a small but significant correlation with NAFLD fibrosis score and no correlation with Fib-4. There are several reasons why it is difficult to compare a new parameter with these index scores: Both Fib-4 and the NAFLD fibrosis score have been shown to have unacceptable diagnostic accuracy among non-obese individuals as well as in subjects aged $\leq 35$ or $>65$ years, making them difficult to interpret in our material $[29,30]$. That being said, they both perform best when used to rule out, not rule in, advanced fibrosis, and none of our subjects were above the upper cutoff value, where an excellent specificity has been demonstrated [31].

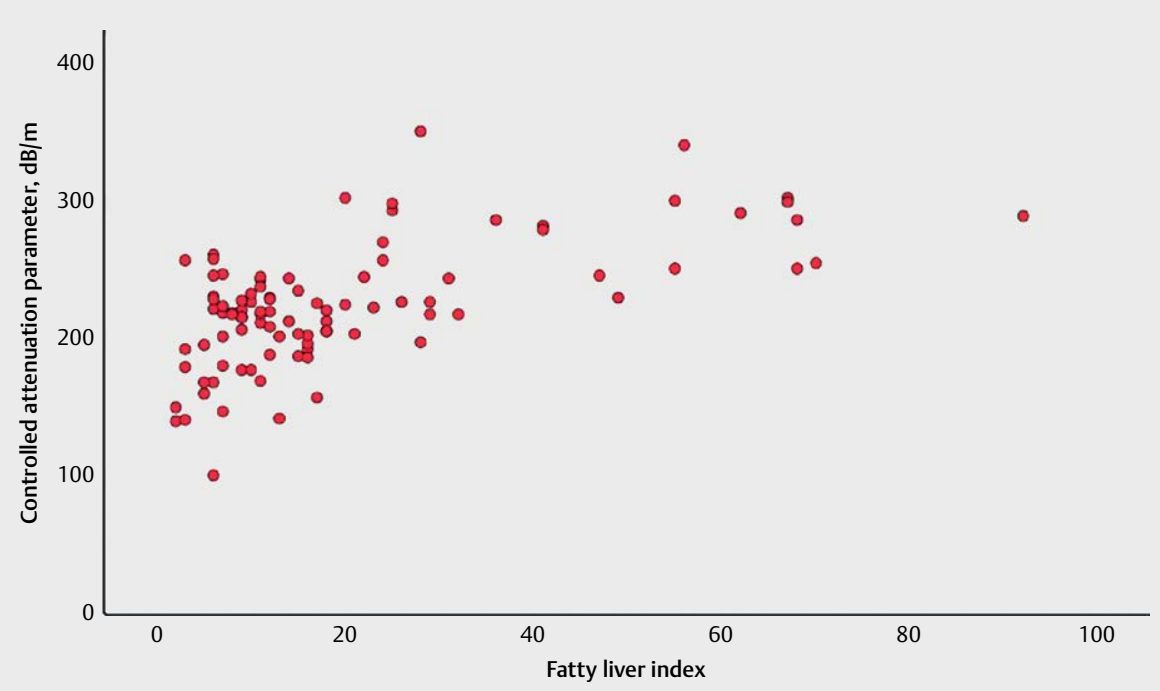

- Fig. 5 Controlled attenuation parameter (CAP) is significantly correlated with the fatty liver index in adults (rho $0.55, \mathrm{p}<0.001$ ). 


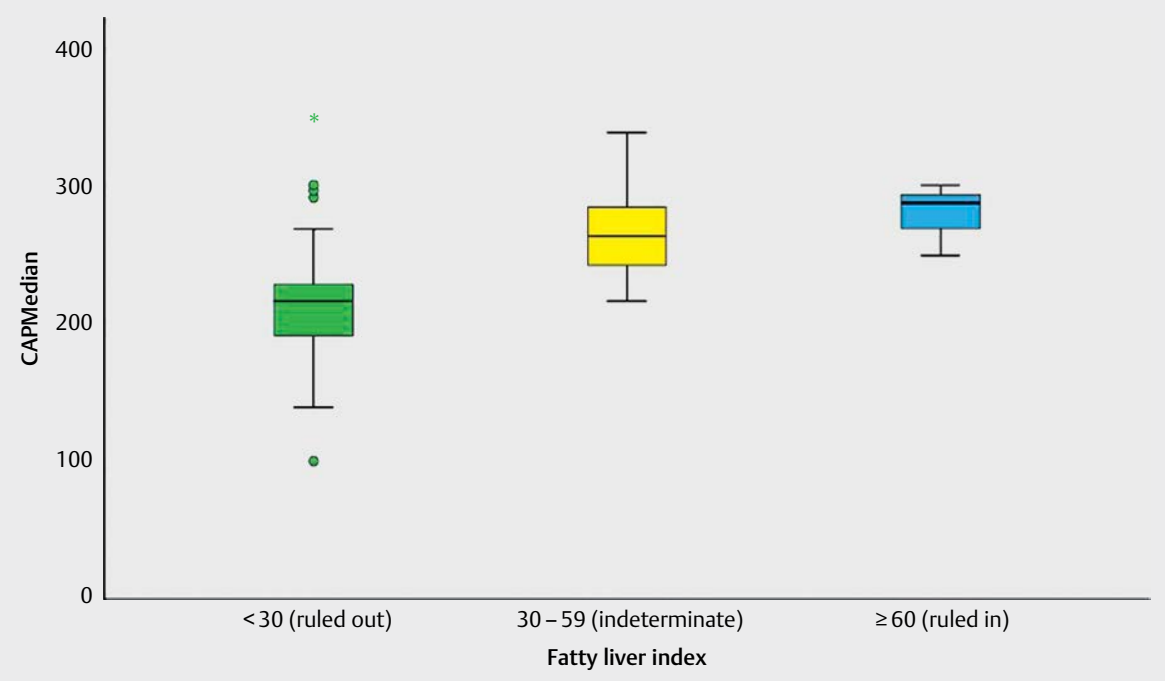

Fig. 6 Controlled attenuation parameter (CAP) for low, moderate, and high fatty liver index (FLI). FLI: <30 (ruled out); 30-59 (indeterminate); $\geq 60$ (ruled in).

- Table 3 Correlation between CAP and clinical features for children.

\begin{tabular}{|l|l|l|}
\hline Clinical features & Correlation (r) & p-value \\
\hline Waist-to-height ratio & 0.292 & 0.008 \\
\hline BMl z-score & 0.271 & 0.014 \\
\hline Waist circumference z-score & 0.116 & n.s. \\
\hline Skin-to-capsule distance & 0.057 & n.s. \\
\hline
\end{tabular}

CAP correlated with age in adults but not in children. In linear regression, we found that the increasing CAP values during adulthood only reflected the increasing prevalence of obesity with age. CAP values were higher in adults than in children. This difference persisted after correction for the increased BMI in adulthood, suggesting that CAP increase may be a phenomenon linked to the transition into adulthood, as there was no significant effect of age within the two individual cohorts. This is in contrast to liver stiffness measurements, where an age effect is often found in children [15].

The use of quality criteria is heavily advocated in liver stiffness measurements, particularly the use of IQR/M. There is no consensus on the use of similar criteria in CAP measurements. However, it was reported that IQR seems to be independent of CAP values and that an IQR $<40$ increases the diagnostic accuracy of CAP [12]. Other authors have not been able to reproduce this while arguing that a low IQR/M for CAP significantly increases the accuracy of diagnosing steatosis [13], with several suggested cutoff values: $<0.1,<0.2$, and $<0.3$.

In our study, we observe that a substantial number of subjects do not meet the proposed quality criteria, with an IQR $<40$ in $45 \%$ and $61 \%$, and an $\mathrm{IQR} / \mathrm{M}<0.1$ in $9 \%$ and $28 \%$, in children and adults, respectively.
Our findings support a lack of correlation between CAP and IQR value, meaning that measurement variation is similar for a range of CAP values. Thus, with a stable IQR value across CAP values, the $\mathrm{IQR} / \mathrm{M}$ will decrease when the CAP increases. In our cohort of healthy individuals, it is impossible to evaluate the effect of IQR or $\mathrm{IQR} / \mathrm{M}$ on diagnostic accuracy.

Although already obviously useful, CAP will hopefully continue to improve, with promising modifications, such as continuous CAP, in the pipeline [32].

\section{Limitations}

The major limitation is the lack of liver biopsies or magnetic resonance imaging-based fat fraction quantification (MRI-PDFF), making us unable to rule out liver steatosis with certainty. We tried to combat this by excluding obese individuals and performing B-mode ultrasound to rule out increased liver echogenicity. We performed blood tests to exclude other liver diseases in adults, but it was deemed unethical in healthy children.

\section{Conclusion}

CAP is highly correlated to BMI z-score across all ages, even in subjects with a BMI below average (z-score $<0)$. Skin-to-capsule distance in adults is a stronger predictor of increased CAP compared to BMI. Approximately 1 in 5 healthy, non-obese subjects had a CAP value above the suggested cutoffs for liver steatosis, warranting further research to clarify whether this should lead to an adjustment of reference values. Using our results, CAP ULN would be increased from 225 and 248 to 253 and 295 in children and adults, respectively. 


\section{Conflict of Interest}

The authors declare that they have no conflict of interest.

\section{References}

[1] Younossi ZM, Koenig AB, Abdelatif D et al. Global Epidemiology of Nonalcoholic Fatty Liver Disease-Meta-Analytic Assessment of Prevalence, Incidence, and Outcomes. Hepatology 2016; 64: 73-84

[2] Vos MB, Abrams SH, Barlow SE et al. NASPGHAN Clinical Practice Guideline for the Diagnosis and Treatment of Nonalcoholic Fatty Liver Disease in Children: Recommendations from the Expert Committee on NAFLD (ECON) and the North American Society of Pediatric Gastroenterology. Hepatology and Nutrition (NASPGHAN). J Pediatr Gastr Nutr 2017; 64: 319-334

[3] Selvakumar PKC, Kabbany MN, Lopez R et al. Prevalence of Suspected Nonalcoholic Fatty Liver Disease in Lean Adolescents in the United States. J Pediatr Gastr Nutr 2018; 67: 75-79

[4] Zou B, Yeo YH, Nguyen VH et al. Prevalence, characteristics and mortality outcomes of obese, nonobese and lean NAFLD in the United States, 1999-2016. J Intern Med 2020; 288: 139-151

[5] Chalasani N, Younossi Z, Lavine JE et al. The diagnosis and management of nonalcoholic fatty liver disease: Practice guidance from the American Association for the Study of Liver Diseases. Hepatology 2018; 67: 328-357

[6] Hernaez R, Lazo M, Bonekamp S et al. Diagnostic Accuracy and Reliability of Ultrasonography for the Detection of Fatty Liver: A Meta-Analysis. Hepatology 2011; 54: 1082-1090

[7] von Volkmann HL, Havre RF, Loberg EM et al. Quantitative measurement of ultrasound attenuation and Hepato-Renal Index in Non-Alcoholic Fatty Liver. Med Ultrason 2013; 15: 16-22

[8] Sasso M, Beaugrand M, de Ledinghen V et al. Controlled Attenuation Parameter (Cap): A Novel Vcte (Tm) Guided Ultrasonic Attenuation Measurement for the Evaluation of Hepatic Steatosis: Preliminary Study and Validation in a Cohort of Patients with Chronic Liver Disease from Various Causes. Ultrasound in Medicine and Biology 2010; 36: $1825-1835$

[9] Karlas T, Petroff D, Sasso M et al. Individual patient data meta-analysis of controlled attenuation parameter (CAP) technology for assessing steatosis. Journal of Hepatology 2017; 66: 1022-1030

[10] Desai NK, Harney S, Raza R et al. Comparison of Controlled Attenuation Parameter and Liver Biopsy to Assess Hepatic Steatosis in Pediatric Patients. J Pediatr-Us 2016; 173: 160-164.e1

[11] Pu K, Wang YP, Bai SY et al. Diagnostic accuracy of controlled attenuation parameter (CAP) as a non-invasive test for steatosis in suspected non-alcoholic fatty liver disease: A systematic review and meta-analysis. Bmc Gastroenterol 2019; 19: 51

[12] Wong VW, Petta S, Hiriart JB et al. Validity criteria for the diagnosis of fatty liver by $\mathrm{M}$ probe-based controlled attenuation parameter. J Hepatol 2017; 67: 577-584

[13] Semmler G, Woran K, Scheiner B et al. Novel reliability criteria for controlled attenuation parameter assessments for non-invasive evaluation of hepatic steatosis. United European. Gastroenterol J 2020; 8: $321-331$

[14] Mulabecirovic A, Mjelle AB, Gilja $\mathrm{OH}$ et al. Liver elasticity in healthy individuals by two novel shear-wave elastography systems-Comparison by age, gender, BMI and number of measurements. PLoS One 2018; 13: e0203486
[15] Mjelle AB, Mulabecirovic A, Havre RF et al. Normal Liver Stiffness Values in Children: A Comparison of Three Different Elastography Methods. J Pediatr Gastroenterol Nutr 2019; 68: 706-712

[16] Bedogni G, Bellentani S, Miglioli L et al. The Fatty Liver Index: a simple and accurate predictor of hepatic steatosis in the general population. Bmc Gastroenterol. 2006; 6: 33

[17] Angulo P, Hui JM, Marchesini G et al. The NAFLD fibrosis score: A noninvasive system that identifies liver fibrosis in patients with NAFLD. Hepatology 2007; 45: 846-854

[18] Sterling RK, Lissen E, Clumeck $N$ et al. Development of a simple noninvasive index to predict significant fibrosis in patients with HIV/ HCV coinfection. Hepatology 2006; 43: 1317-1325

[19] Shah AG, Lydecker A, Murray K et al. Comparison of Noninvasive Markers of Fibrosis in Patients With Nonalcoholic Fatty Liver Disease. Clin Gastroenterol H 2009; 7: 1104-1112

[20] Angulo P, Bugianesi E, Bjornsson ES et al. Simple Noninvasive Systems Predict Long-term Outcomes of Patients With Nonalcoholic Fatty Liver Disease. . Gastroenterology 2013; 145: 782-9.e4

[21] Kaya E, Demir D, Alahdab YO et al. Prevalence of hepatic steatosis in apparently healthy medical students: a transient elastography study on the basis of a controlled attenuation parameter. Eur J Gastroenterol Hepatol 2016; 28: 1264-1267

[22] de Ledinghen V, Vergniol J, Capdepont M et al. Controlled attenuation parameter (CAP) for the diagnosis of steatosis: a prospective study of 5323 examinations. J Hepatol 2014; 60: 1026-1031

[23] Younossi Z, Anstee QM, Marietti M et al. Global burden of NAFLD and NASH: trends, predictions, risk factors and prevention. Nat Rev Gastroenterol Hepatol 2018; 15: 11-20

[24] Yilmaz Y, Kani HT, Demirtas CO et al. Growing burden of nonalcoholic fatty liver disease in Turkey: A single-center experience. Turk Gastroenterol 2019; 30: 892-898

[25] Denkmayr L, Feldman A, Stechemesser L et al. Lean Patients with Non-Alcoholic Fatty Liver Disease Have a Severe Histological Phenotype Similar to Obese Patients. J Clin Med 2018; 7: 562

[26] Akyuz U, Yesil A, Yilmaz Y.. Characterization of lean patients with nonalcoholic fatty liver disease: potential role of high hemoglobin levels. Scand J Gastroenterol 2015; 50: 341-346

[27] Vanderwall C, Clark RR, Eickhoff ] et al. BMI is a poor predictor of adiposity in young overweight and obese children. Bmc Pediatr 2017; 17: 135

[28] Carvalhana S, Leitao J, Alves AC et al. How good is controlled attenuation parameter and fatty liver index for assessing liver steatosis in general population: correlation with ultrasound. Liver Int 2014; 34: e111-117

[29] Eren F, Kaya E, Yilmaz Y.. Accuracy of Fibrosis-4 index and non-alcoholic fatty liver disease fibrosis scores in metabolic (dysfunction) associated fatty liver disease according to body mass index: failure in the prediction of advanced fibrosis in lean and morbidly obese individuals. Eur J Gastroenterol Hepatol 2020; DOI: 10.1097| MEG.0000000000001946

[30] McPherson S, Hardy T, Dufour JF et al. Age as a Confounding Factor for the Accurate Non-Invasive Diagnosis of Advanced NAFLD Fibrosis. Am J Gastroenterol 2017; 112: 740-751

[31] Kaya E, Bakir A, Kani HT et al. Simple Noninvasive Scores Are Clinically Useful to Exclude, Not Predict, Advanced Fibrosis: A Study in Turkish Patients with Biopsy-Proven Nonalcoholic Fatty Liver Disease. Gut Liver 2020; 14: 486-491

[32] Audière S, Fournier C, Whitehead J et al. Continuous CAP algorithm: reduced variability in a prospective cohort. EASL - The digital international liver congress. J Hepatol 2020; 73: S436 